\title{
Mutations in lipid transporter ABCA12 in harlequin ichthyosis and functional recovery by corrective gene transfer
}

\author{
Masashi Akiyama, ${ }^{1}$ Yoriko Sugiyama-Nakagiri, ${ }^{1}$ Kaori Sakai, ${ }^{1}$ James R. McMillan,, ${ }^{2}$ Maki Goto, ${ }^{1}$ \\ Ken Arita, ${ }^{1}$ Yukiko Tsuji-Abe, ${ }^{1}$ Nobuko Tabata, ${ }^{3}$ Kentaro Matsuoka, ${ }^{4}$ Rikako Sasaki, ${ }^{5}$ \\ Daisuke Sawamura, ${ }^{1}$ and Hiroshi Shimizu${ }^{1}$ \\ 1Department of Dermatology, Hokkaido University Graduate School of Medicine, Sapporo, Japan. ${ }^{2}$ Creative Research Initiative Sousei, Hokkaido University, \\ Sapporo, Japan. ${ }^{3}$ Division of Dermatology, Japanese Red Cross Sendai Hospital, Yagiyama, Tashiro, Sendai, Japan. ${ }^{4}$ Division of Pathology and \\ ${ }^{5}$ Division of Dermatology, National Center for Child Health and Development, Okura, Setagaya, Tokyo, Japan.
}

\begin{abstract}
Harlequin ichthyosis (HI) is a devastating skin disorder with an unknown underlying cause. Abnormal

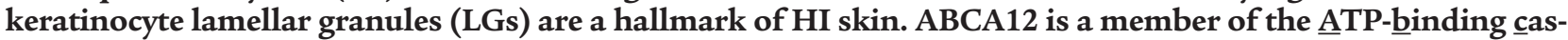
sette transporter family, and members of the ABCA subfamily are known to have closely related functions as lipid transporters. ABCA3 is involved in lipid secretion via LGs from alveolar type II cells, and missense mutations in $A B C A 12$ have been reported to cause lamellar ichthyosis type 2, a milder form of ichthyosis. Therefore, we hypothesized that HI might be caused by mutations that lead to serious ABCA12 defects. We identify 5 distinct $A B C A 12$ mutations, either in a compound heterozygous or homozygous state, in patients from $4 \mathrm{HI}$ families. All the mutations resulted in truncation or deletion of highly conserved regions of ABCA12. Immunoelectron microscopy revealed that ABCA12 localized to LGs in normal epidermal keratinocytes. We confirmed that ABCA12 defects cause congested lipid secretion in cultured HI keratinocytes and succeeded in obtaining the recovery of $L G$ lipid secretion after corrective gene transfer of $A B C A 12$. We concluded that ABCA12 works as an epidermal keratinocyte lipid transporter and that defective ABCA12 results in a loss of the skin lipid barrier, leading to HI. Our findings not only allow DNA-based early prenatal diagnosis but also suggest the possibility of gene therapy for $\mathrm{HI}$.
\end{abstract}

\section{Introduction}

During the evolutionary process, when our ancestors left the safety of the aquatic environment, they developed a robust, protective mechanism or process in the skin that allowed adjustment to the new, dry environment; this is now known as keratinization. In humans, congenital defects involving skin keratinization cause a unique genodermatosis, ichthyosis $(1,2)$, which was named after the Greek word ichthys, meaning fish. Among the variety of types of ichthyosis, harlequin ichthyosis (HI) (Mendelian Inheritance of Man [MIM] 242500) is the most serious subtype (Figure 1); it is also the most severe congenital skin disorder of unknown etiology.

ABCA12 belongs to a large superfamily of ATP-binding cassette $(\mathrm{ABC})$ transporters, which aid in the transport of various biomolecules across the cell membrane (3-5). The ABCA subfamily is thought to be important in lipid transport (6). ABCA12 is phylogenetically related to $\mathrm{ABCA}$, which is essential for alveolar surfactant lipid transport/secretion by lamellar granules (LGs) in type II alveolar lung cells. In skin, LGs are the most common secretory granules present in upper epidermal keratinocytes (7). Abnormal LGs are the most obvious characteristic findings in HI lesional epidermis. Furthermore, relatively minor missense mutations in $A B C A 12$ have been reported to underlie type 2 lamellar ichthyosis (MIM 601277), a milder form of ichthyosis (8). Thus, we hypoth-

Nonstandard abbreviations used: ABC, ATP-binding cassette; CT, threshold cycle; HI, harlequin ichthyosis; LG, lamellar granule; MIM, Mendelian Inheritance of Man. Conflict of interest: The authors have declared that no conflict of interest exists.

Citation for this article: J. Clin. Invest. 115:1777-1784 (2005).

doi:10.1172/JCI24834. esized that $A B C A 12$ mutations leading to serious $\mathrm{ABCA12}$ protein defects might underlie HI.

In the present study, we demonstrate that ABCA12 works as an epidermal keratinocyte lipid transporter and that defective $\mathrm{ABCA} 12$ results in a loss of the skin lipid barrier, leading to HI. We have found 5 distinct truncation, deletion, or splice-site mutations in 4 independent $\mathrm{HI}$ families. These mutations were present on both alleles of our HI patients, either in compound heterozygous or homozygous state. We demonstrate that ABCA12 mRNA is expressed in normal human keratinocytes and that this expression is upregulated during keratinization. Our immunoelectron microscopic findings showed that ABCA12 protein localizes to LGs in the upper epidermal keratinocytes of human skin. Ultrastructural and immunofluorescent examination of human skin and cultured epidermal keratinocytes from HI patients who harbor $A B C A 12$ mutations revealed defective lipid secretion of LG lipid contents. In addition, using $A B C A 12$ corrective gene transfer in cultured $\mathrm{HI}$ keratinocytes, we have succeeded in restoring the normal ability of HI cells to secrete LG lipid.

\section{Results}

ABCA12 expression and localization in epidermal keratinocytes. To confirm the expression of $A B C A 12$ mRNA in human epidermal keratinocytes, we performed semiquantitative RT-PCR assays and demonstrated strong expression of $A B C A 12$ transcripts in cultured normal epidermal keratinocytes. To investigate the upregulation of $A B C A 12$ during keratinization under high- $\mathrm{Ca}^{2+}$ conditions, we evaluated the $A B C A 12$ transcript population against the GAPDH transcript using real-time RT-PCR. Calculation of the expres- 


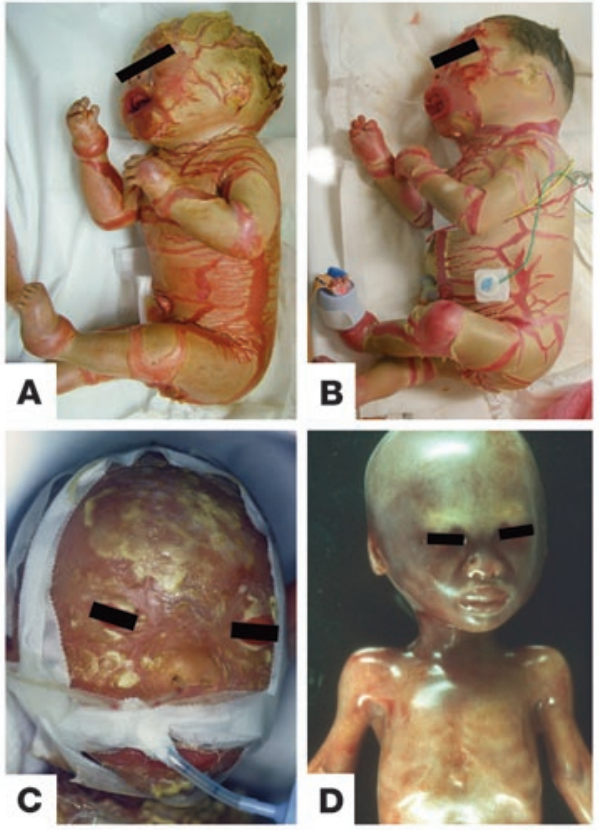

sion ratios showed that $A B C A 12$ transcription was upregulated 4-fold after 1 week in high-Ca ${ }^{2+}$ culture. Furthermore, to confirm the expression of $\mathrm{ABCA} 12$ protein in the epidermis, we raised a polyclonal anti-ABCA12 antibody that recognizes an epitope near the $\mathrm{C}$ terminus of the ABCA12 polypeptide (residues 2567-2580) and performed immunostaining for ABCA12. ABCA12 was positive in the upper epidermal layers, mainly the granular layers, of normal human skin (Figure 2A); in contrast, there was an absence of immunolabeling in epidermal keratinocytes from $\mathrm{HI}$ patient 4 (Figure 2B), who harbors the homozygous truncation mutation

\section{Figure 1}

Clinical features of $\mathrm{HI}$ patients. (A) Patient 1 from family $\mathrm{A}$ harboring a homozygous mutation IVS23-2A $\rightarrow \mathrm{G}$ in $A B C A 12$. (B) Patient 2 from family $B$ with compound heterozygous $A B C A 12$ mutations, IVS23-2A $\rightarrow$ G and 5848C $\rightarrow$ T (R1950X). (C) Patient 3 (family C) carrying compound heterozygous $A B C A 12$ mutations, 2021_2022del AA and 4158_4160delTAC (T1387del). (D) An affected fetus from family $C$ aborted at 23 weeks' gestation showed no serious symptoms, although some abnormal keratinization was observed mainly on the cheeks and the perioral area.

$\mathrm{R} 434 \mathrm{X}$ (see below). This mutation resulted in truncation of the protein, leading to loss of the epitope for the anti-ABCA12 antibody. These findings confirmed specificity of the anti-ABCA12 antibody. In the epidermis of patient 1, who harbors a homozygous splice acceptor site mutation IVS23-2A $\rightarrow$ G (see below), weak ABCA12 immunostaining was seen in the granular layer keratinocytes (Figure $2 \mathrm{C}$ ). Immunoelectron microscopy revealed that ABCA12 protein was restricted to the LGs in the cytoplasm of epidermal keratinocytes (Figure 2, D and E). ABCA12-positive LGs were abundant close to the cell membrane and were observed fusing with the cell membrane to secrete their lipid content to the extracellular space of the stratum corneum (Figure $2 \mathrm{~F}$ ). These results indicate that ABCA12 is expressed in keratinocytes during keratinization and is likely to be involved in lipid transport into the extracellular space via LGs to form the stratum corneum lipid barrier (Figure $2 \mathrm{G}$ ).

ABCA12 mutations in HI families. Full-length ABCA12 protein comprises 2595 amino acids and includes $2 \mathrm{ABCs}$ containing 3 characteristic, highly conserved motifs (Walker A, Walker B, and active transport signature). In addition, there are 2 transmembrane domains, each consisting of 6 hydrophobic membrane-spanning helices. Mutational analysis of the 53 exons, including the exonintron boundaries of the entire $A B C A 12$ gene, revealed 5 novel, distinct mutations in both alleles, either in compound heterozygous

\section{Figure 2}

Localization and structure of $A B C A 12$ protein and the sites of $\mathrm{HI}$ mutations. (A) ABCA12 protein (green) was localized in the cytoplasm of the upper epidermal keratinocytes (arrows) in healthy skin. (B) No ABCA12 immunolabeling was seen in the epidermis of patient 4. (C) Weak ABCA12 staining (arrows) was observed in the epidermis of patient 1. Asterisks indicate nonspecific staining in the stratum corneum. Red, nuclear counterstain. Scale bar: $10 \mu \mathrm{m}$. (D-F) By immunoelectron microscopy, ABCA12 protein $(5 \mathrm{~nm}$ gold particles) was restricted to LGs (arrows) in the upper epidermal cell (D). Lamellar structures were apparent in some ABCA12-positive LGs (E). ABCA12-positive LGs (arrows) were observed close to the keratinocytecell membrane (white circles) and they fused with it to secrete their contents into the intercellular space $(\mathbf{F})$. Scale bars: $0.2 \mu \mathrm{m}$. (G) Model of ABCA12 function in the skin. ABCA12 transports lipid into the $L G$, and ABCA12-positive LGs fuse with the cell membrane to secrete lipid into extracellular space to form the intercellular lipid layer. (H) Structure of ABCA12 protein and the 5 mutation sites (red arrows) in $\mathrm{HI}$ families. Dark-blue area, cell membrane; bottom of dark-blue area, cytoplasmic surface.
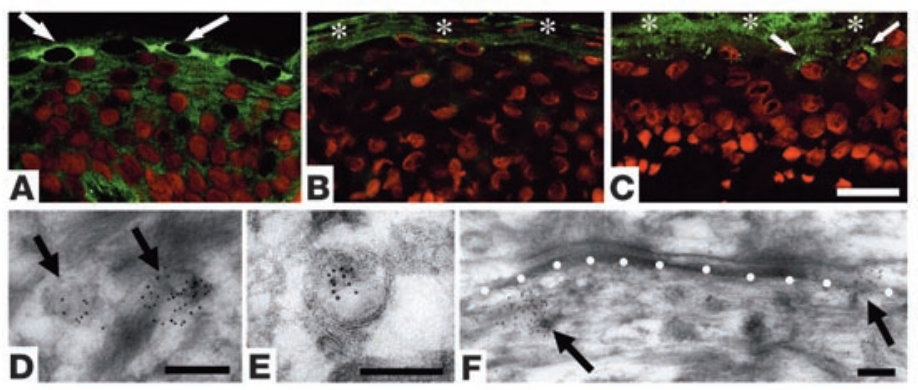

G

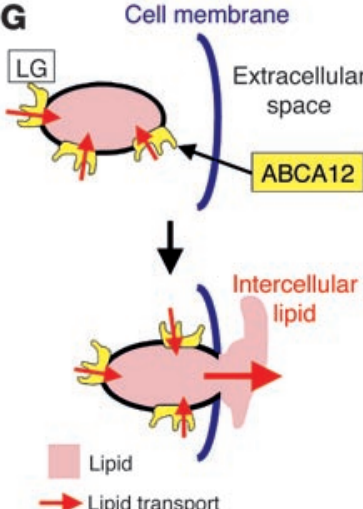

H IVS23-2A $\rightarrow$ G (intron23) R1950X (exon39)

R1950X (exon39)

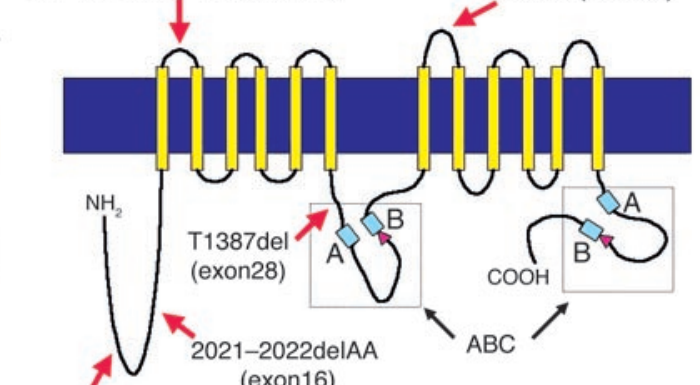

R434X (exon12) (exon16)
Transmembrane domain Walker A and B motifs 4 Active transport signature 
or homozygous state, in all patients from $4 \mathrm{HI}$ families (Figure $2 \mathrm{H})$. The mutations in patients were homozygous in the 2 consanguineous families and compound heterozygous in the 2 nonconsanguineous families (Figure 3 ). The mutations were verified in the heterozygous parents. Each of the three mutations, $1300 \mathrm{C} \rightarrow \mathrm{T}$ $(\mathrm{R} 434 \mathrm{X}), 2021 \_2022$ delAA, and 5848C $\rightarrow \mathrm{T}$ (R1950X), resulted in truncation of a highly conserved region of the ABCA12 protein. The deletion mutation 4158_4160delTAC led to an in-frame deletion of a highly conserved threonine residue at codon 1387 (T1387del) within the first ATP-binding domain of the ABCA12 protein (Figure 4D). A splice acceptor site mutation, IVS23-2A $\rightarrow \mathrm{G}$, was verified by RT-PCR in mRNA from the patient's cultured keratinocytes (Figure 4, A-C). RT-PCR products from the patient showed 2 splice pattern variants different from the normal splicing variant in which 1 mutant transcript loses a 9-bp sequence from exon 24, which results in a 3 -amino acid deletion (Y1099_K1101del). These 3 amino acids are located between the transmembrane domains and are highly conserved (Figure 4D). The other mutant transcript lost a $170-b p$ sequence from exon 24 , which led to a frameshift. All of these mutations are thought to seriously affect either the function or specific critical structures of the ABCA12 protein.

Disturbed lipid secretion in epidermis of HI patients. Morphological observations revealed extraordinarily thick stratum corneum (Figure 5A) and abnormal LG secretion in keratinocytes of the epidermis of HI patients (Figure 5, C and E). Ultrastructurally, the cytoplasm in the stratum corneum was congested with abnormal lipid-containing droplets and vacuoles that resembled immature LG-like vesicles. In the keratinocyte cytoplasm of the granular layer, no normal LGs were apparent.

Immunofluorescent staining showed that glucosylceramide, a major lipid component of LG $(7,9)$ and an essential component of the epidermal permeability barrier (10), was diffusely distributed throughout the epidermis of HI patients (Figure 5G); this contrasts with the restricted, intense distribution in the stratum corneum of healthy skin (Figure 5H).

Abnormal lipid secretion in keratinocytes of HI patients and recovery of ABCA12 function by corrective gene transfer. Keratinocytes from patient 1 , cultured under high-Ca ${ }^{2+}$ conditions $(2.0 \mathrm{mM})$, expressed only a small amount of mutated ABCA12 protein (Figure 6J). Culture of HI

C

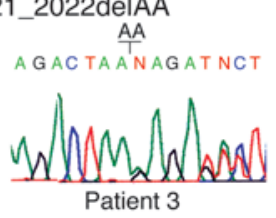

Figure 3 keratinocytes under high- $\mathrm{Ca}^{2+}$ conditions $(2.0 \mathrm{mM})$ induced a large number of cells to exhibit intense glucosylceramide staining around the nuclei, and this glucosylceramide failed to localize to the periphery of the keratinocyte cytoplasm (congested pattern) (Figure 6, A and C). Conversely, culture of healthy keratinocytes in high- $\mathrm{Ca}^{2+}$ conditions resulted in a large proportion of cells with diffuse glucosylceramide staining throughout the cytoplasm (widely-distributed pattern) (Figure 6, B and D). This difference was most pronounced after 1 week in culture (Figure $6, \mathrm{C}$ and D). Electron microscopic observation of HI keratinocytes cultured for 1 week in high- $\mathrm{Ca}^{2+}$ conditions revealed that LGs formed, although proper secretion of their contents was not observed (Figure 6E). This finding suggests defects in LG lipid transport. Double immunostaining for ABCA12 protein
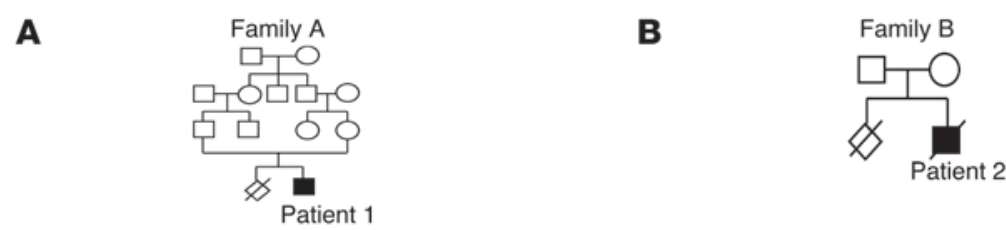

IVS23-2A $\rightarrow$ G (homozygote)

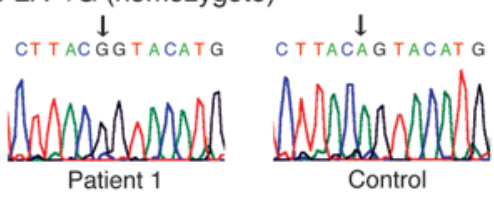

IVS23-2A $\rightarrow G_{\downarrow}$
CTTACNGTACATG
$\stackrel{\downarrow}{\downarrow}$ CT TACAGTACATG

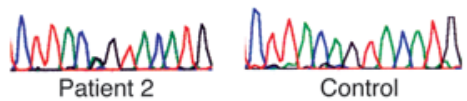

$5848 \mathrm{C} \rightarrow \mathrm{T}(\mathrm{R} 1950 \mathrm{X})$
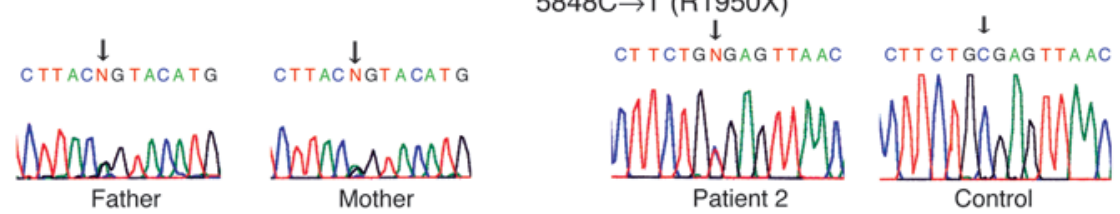

Family $\mathrm{D}$

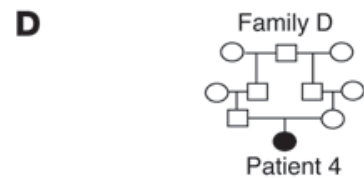

$1300 \mathrm{C} \rightarrow \mathrm{T}(\mathrm{R} 434 \mathrm{X})$ (homozygote)
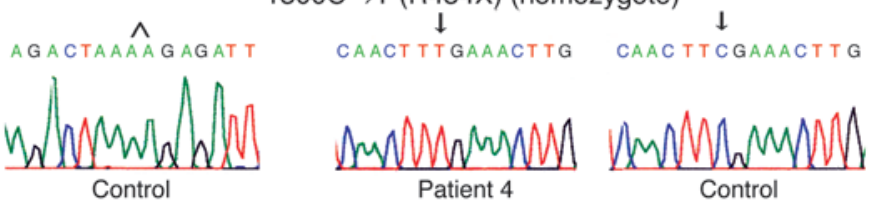

Contro

4158_4160delTAC (T1387del)
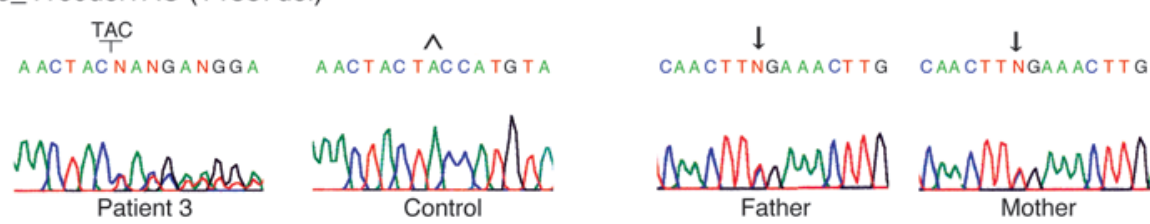

Families with $\mathrm{HI}$ and $A B C A 12$ mutations. (A) Patient 1 from family $\mathrm{A}$ was a homozygote for the mutation IVS23-2A $\rightarrow G$, and both his parents were heterozygous carriers. (B) Patient 2 from family $B$ was a compound heterozygote for the mutations IVS23-2A $\rightarrow$ G and 5848C $\rightarrow$ T (R1950X). (C) Patient 3 from family $C$ was a compound heterozygote for the mutations 2021_2022delAA and 4158_4160delTAC (T1387del). (D) Patient 4 from family D was a homozygote for the mutation $1300 \mathrm{C} \rightarrow \mathrm{T}(\mathrm{R} 434 \mathrm{X})$, and her 2 parents were heterozygous carriers. 


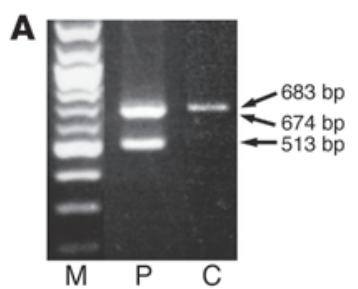

B CT TCATG AGT ACATG A AG ATG

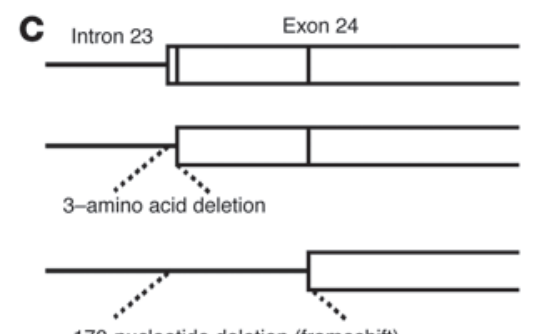

170-nucleotide deletion (frameshift)
Normal transcript

Mutant transcript

(3-amino acid deletion)

(Y1099_K1101 del)

Mutant transcript

(170-nucleotide deletion)

(frameshift)

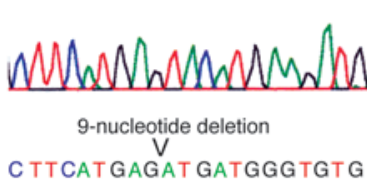

C TTCATGAGAT GATGGGTGTG

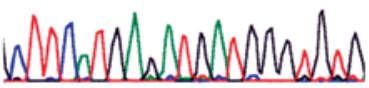

170-nucleotide deletion

CTTCATGAGCTTCTCGGTTAT

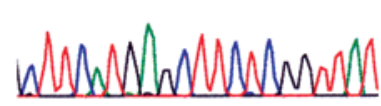

Normal transcript

Mutant transcript

(3-amino acid deletion)

Mutant transcript

(170-nucleotide deletion)

\section{D}

IVS23-2A $\rightarrow$ G: Y1099_K1101del

Homo sapiens

Rattus norvegicus

Mus musculus

Gallus gallus

Caenorhabditis elegans*

1089VYEKDLRLHEYMKMMGVNSCSHF1111

VYEKDLRLHEYMKMMGVNSCSHF

VYEKDLRLHEYMKMMGVNSCSHF

VQEKDLRLYEYMKMMGVNASSHF

VVEKEDRLKEYMRVMG+N +HF

4158_4160delTAC: T1387del

$\begin{array}{lc}\text { Homo sapiens } & \text { 1373ITSLLGPNGAGKTTTISMLTGLFGASAG1400 } \\ \text { Rattus norvegicus } & \text { ITSLLGPNGAGKTTTISMLTGLFGATAG } \\ \text { Mus musculus } & \text { ITSLLGPNGAGKTTTMYVIFQLNVKEGY } \\ \begin{array}{l}\text { Gallus gallus } \\ \text { Caenorhabditis elegans* }\end{array} & \text { ITSLLGHNGAGTTTISILTGLFPTSSG } \\ \text { CTVLLGHNGAGKSTTFSMLTGVASPSSG }\end{array}$

Figure 4

Verification of splice-site mutation IVS23-2A $\rightarrow$ G and conservation of residues deleted by mutations IVS23-2A $\rightarrow$ G and 4158_4160delTAC (T1387del). (A) RT-PCR analysis of mRNA fragments around the exon 23-24 boundary indicated that keratinocytes from patient 1 (lane $P$ ) showed 2 different mutant transcripts, $674 \mathrm{bp}$ and $513 \mathrm{bp}$, which were shorter than the control transcript (683 bp) from healthy human keratinocytes (lane C). Lane M, markers. (B) Sequencing of the mutant transcripts and the control transcript revealed that 9 nucleotides and 170 nucleotides were deleted in mutant transcripts. (C) A 9-nucleotide deletion resulted in the loss of 3 amino acids from the $\mathrm{N}$ terminal sequence of exon 24 (Y1099_K1101del), and the 170-nucleotide deletion led to a frameshift. (D) ABCA12 amino acid sequence alignment shows the level of conservation in diverse species of the amino acids, Y1099_K1101 and T1387 (red characters), which were deleted by mutations in HI families. Asterisks indicate ABC (abt-4).

and glucosylceramide clearly demonstrated that, before genetic correction of ABCA12, keratinocytes from patient 1 with a low expression of mutated ABCA12 protein showed a congested glucosylceramide distribution pattern (Figure 6, J-L). After genetic correction, however, the HI patient's keratinocytes, now expressing normal ABCA12, showed a normal, widely-distributed pattern of glucosylceramide staining (Figure 6, M-O). Corrective ABCA12 gene transfer into cultured $\mathrm{HI}$ keratinocytes resulted in a significant increase in number of cells exhibiting the widely-distributed pattern of glucosylceramide staining from $6.98 \% \pm 3.33 \%$ (control nontransfected patients' cells) to $16.70 \% \pm 2.14 \%$ (transfected patients' cells) (Student's $t$ test, $P<0.02$ ) (Figure 6P). These results clearly indicate that an ABCA12 deficiency leads to defective LG lipid transport into the intercellular space in $\mathrm{HI}$ patients, both in the epidermis and in cultured keratinocytes.

\section{Discussion}

An abnormal synthesis or metabolism of the LG lipid contents was previously suspected as being a possible pathogenetic mechanism underlying $\mathrm{HI}(11,12)$. Here, we demonstrate that a severe ABCA12 deficiency causes defective lipid transport via LG in keratinizing epidermal cells, resulting in the HI phenotype.

The ABC transporter superfamily is one of the largest gene families, encoding a highly conserved group of proteins involved in energy-dependent active transport of a variety of substrates across membranes, including ions, amino acids, peptides, carbohydrates, and lipids $(3-5,13)$. ABC transporters have nucleotide-binding folds located in the cytoplasm and utilize energy from ATP to transport substrates across the cell membrane (3-5). ABC genes are widely dispersed throughout the eukaryotic genome and are highly conserved between species (6). The ABCA subfamily, of which the $A B C A 12$ gene is a member, comprises 12 full transporter proteins and 1 pseudogene (ABCA11). The ABCA subclass has received considerable attention (14) because mutations in these genes have been implicated in several human genetic diseases (15-19).

The ABCA1 protein is mutated in the following recessive disorders involving cholesterol and phospholipid transport: Tangier disease (MIM 205400), familial hypoalphalipoproteinemia (MIM 604091), and premature atherosclerosis, depending on the site of the mutations in the protein (17-19). The ABCA4 protein is mutated in Stargardt disease (MIM 248200) as well as in some forms of autosomal recessive retinitis pigmentosa (MIM 601718) and in the majority of cases of autosomal recessive cone-rod dystrophy (MIM 604116), depending on the mutation site or the combination of mutation types $(15,20)$. Heterozygous mutations in $A B C A 4$ have also been implicated in some cases of macular degeneration (MIM $153800)(16,20)$. In a relatively mild type of congenital ichthyosis (lamellar ichthyosis type 2), 5 ABCA12 mutations were reported in 9 families, and all 5 of these mutations were missense mutations that resulted in only 1 amino acid alteration (8). In the present series of HI patients, no $A B C A 12$ missense mutations were identified, and most of the defects led to severe truncation of the ABCA12 peptide, affecting important nucleotide-binding fold domains and/or transmembrane domains. The other, nontrun- 
Patients' skin
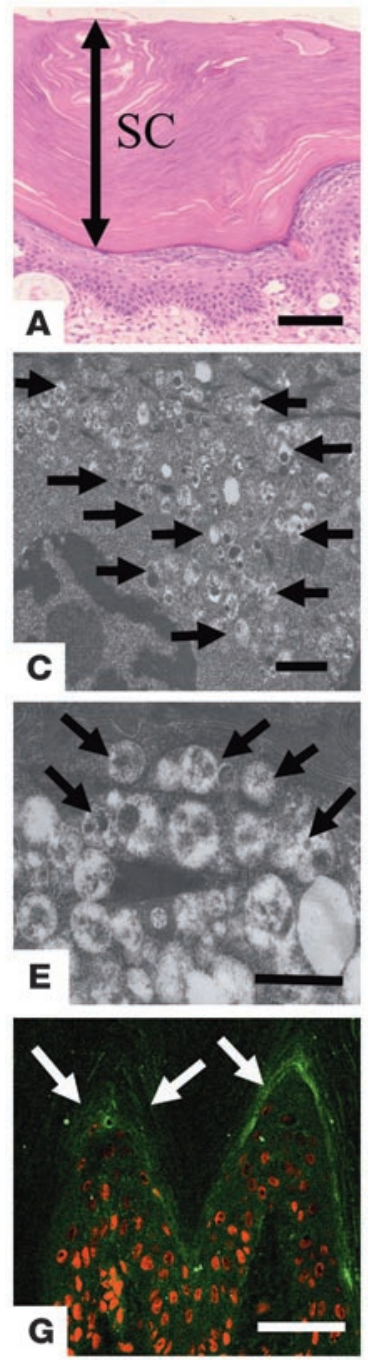

Normal control

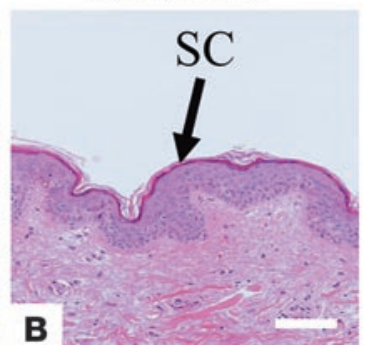

B
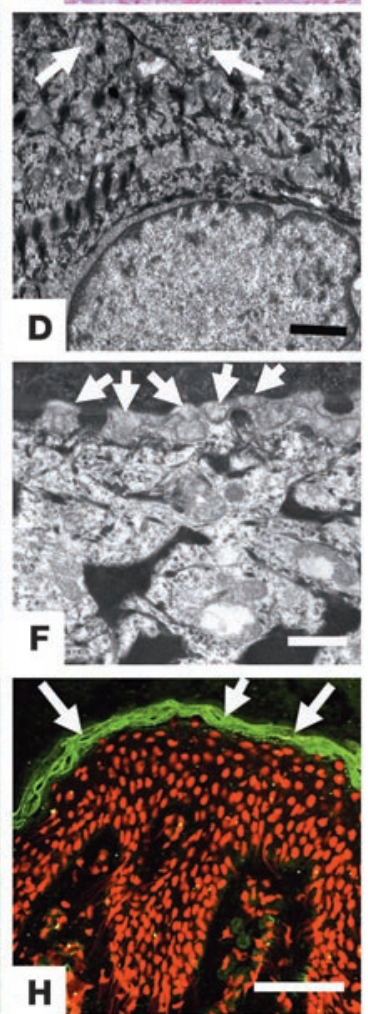

cation mutations in HI were deletion mutations affecting highly conserved $A B C A 12$ sequences (Figure 4D). Thus, it is thought that only truncation or conserved region deletion mutations that seriously affect the function of the ABCA12 transporter protein lead to the HI phenotype. This is in contrast with diseases caused by mutations in other members of the ABCA family. Of ABCA4 mutations causing Stargardt disease, $80 \%$ were missense, and many of these occurred in conserved domains of ABCA4 (21). Of ABCA1 mutations resulting in Tangier disease, $60 \%$ were missense, located within the conserved domains of ABCA1 (22). In this context, $A B C A 12$ mutations underlying $\mathrm{HI}$ are unique in that these mutations are restricted to truncation or deletion mutations.

Patient 1 was homozygous for the splice acceptor site mutation IVS23-2A $\rightarrow$ G. This splice-site mutation was shown to lead to comparable amounts of 2 predicted transcripts, an inframe deletion of 3 amino acids, and a transcript with a 170-nucleotide deletion (frameshift) (Figure 4, A-C). Thus, it is still possible that patient 1 expresses some mutated ABCA12 protein. Indeed, expression of a small amount of ABCA12 protein, although mutated, was detected in the granular cells of the patient's epidermis and cultured keratinocytes by immunofluorescent staining (Figures 2C and 6J).

\section{Figure 5}

Extremely thick stratum corneum and severe disruption of the secretion of LGs in the ABCA12-deficient skin of the present series of $\mathrm{HI}$ patients. (A) Strikingly thick stratum corneum (SC; double arrow) in the patient's skin. (B) Control epidermis showing normal, stratum corneum (arrow). (C) By electron microscopy, LG secretion was disturbed, and many abnormal immature (lacking proper lamellar structures) LGs (arrows) were observed in the keratinocytes. (D) In control skin, LGs (arrows) were distributed in a gradually increasing pattern toward the plasma membrane. (E) Abnormal HI LGs (arrows) were localized close to the cell membrane, but not secreted. (F) LGs were secreted into the extracellular space (arrows). (G) Patient's epidermis including stratum corneum (arrows) showed diffuse staining for glucosylceramide (green), a lipid component of LGs. (H) Glucosylceramide staining (green) was restricted and intense in the stratum corneum (arrows) of normal skin. Red, nuclear stain. Scale bars: $50 \mu \mathrm{m}$ (A, B, G, H); $1 \mu \mathrm{m}$ (C, D); $0.5 \mu \mathrm{m}$ (E, F).

This might have some relevance to the fact that patient 1 survived infancy and is still alive.

$\mathrm{ABCA} 1$ and $\mathrm{ABCA} 4$ are suspected transmembrane transporters for intracellular cholesterol/phospholipids (23-25) and protonated $N$-retinylidenephosphatidylethanolamines (26), respectively. $A B C A 2, A B C A 3$, and $A B C A 7 \mathrm{mRNA}$ levels have been reported to be upregulated by sustained cholesterol influx mediated by modified low-density lipoprotein $(27,28)$, suggesting that ABCA transporters are involved in transmembrane transport of endogenous lipids (23-26). Keratinocyte LGs are known lipid-transporting granules, and LG contents are secreted into the intercellular space, forming an intercellular lipid layer between the granular layer cells and keratinized cells in the stratum corneum, which is essential for the proper barrier function of human skin. Our results indicate that ABCA12 functions in the transport of endogenous lipid across the keratinocyte cell membrane into the stratum corneum intercellular space via LGs. Immunohistological and immunoelectron microscopic observations have indicated that ABCA3 is involved in lipid secretion of pulmonary surfactant in human lung alveolar type II cells (29). ABCA3 and $A B C A 12$ are very closely related in the ABCA subfamily phylogenetic tree (30). It is interesting that the functions of both ABCA3 and ABCA12 are involved in alveolar surfactant and stratum corneum lipid secretion, respectively. This suggests that these 2 transporter systems are evolutionary adaptations to aid the respiratory system and the integument in a dry environment, developed as vertebrates left the aquatic environment and began terrestrial lives. HI skin that harbors serious defects in ABCA12 highlights the important role(s) of epidermal lipid transport in adapting human skin to a terrestrial, dry environment.

$\mathrm{HI}$ is one of the most severe of all genodermatoses and has a very poor prognosis. Thus, parents' request for prenatal diagnosis is to be taken seriously and with care. However, to our knowledge, the causative gene was not identified until now. For the last 20 years, prenatal diagnoses have only been performed by electron microscopic observation of fetal skin biopsy at a late stage of pregnancy (19-23 weeks estimated gestational age) (31-33). In this report, we have identified the causative gene in $\mathrm{HI}$, which now makes possible DNA-based prenatal diagnosis using chorionic villus or amniotic fluid sampling at an earlier stage of pregnancy, with a lower procedural risk and a reduced burden on prospective mothers, similar to that of screening for other severe genetic disorders, including keratinization disorders (34). Furthermore, we have performed correc- 

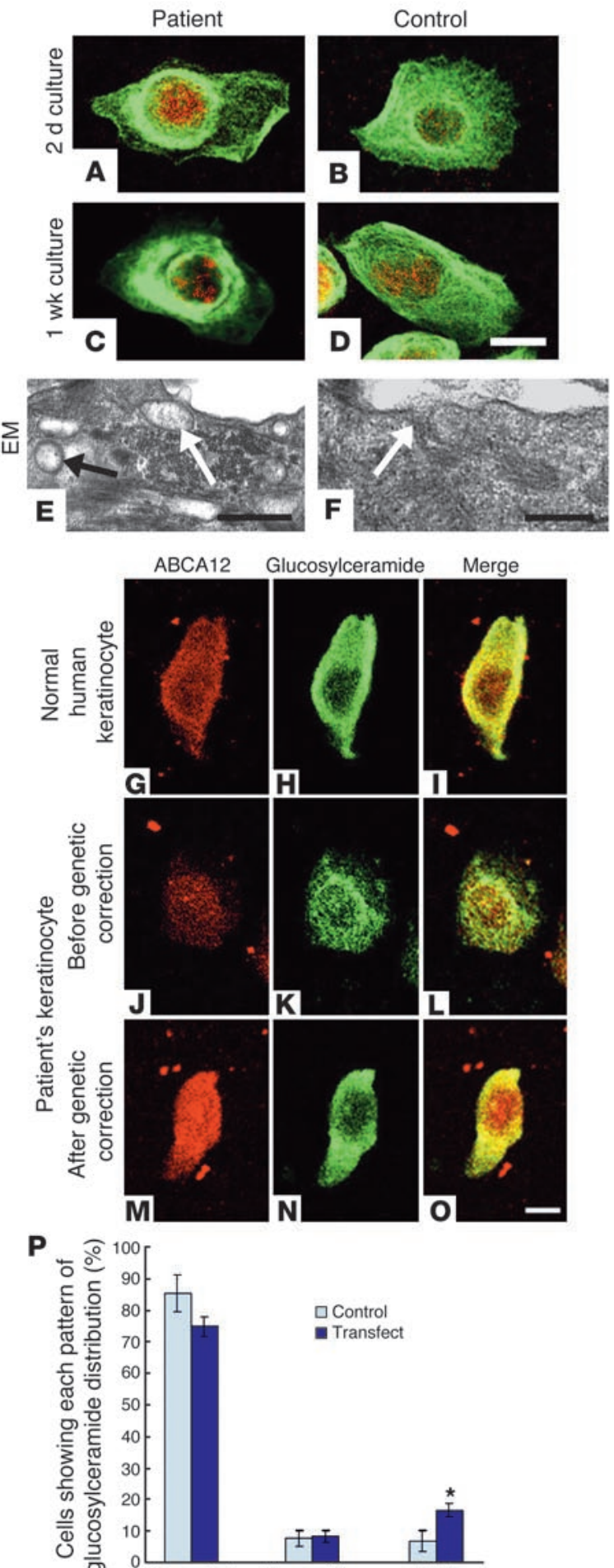

M

N

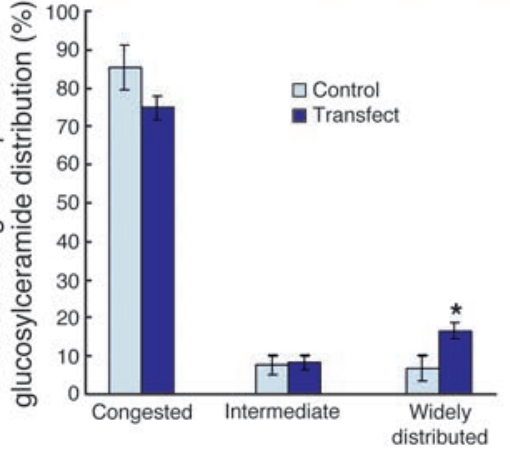

tive gene transfer in $\mathrm{HI}$ keratinocytes and succeeded in obtaining phenotypic rescue of a patient's cultured keratinocytes. These data provide significant clues that establish a strategic approach to HI gene therapy treatment. We believe that the genetic information presented in this study will be highly beneficial to our understanding of HI pathogenesis and in optimizing HI patient diagnosis, genetic counseling, care, and treatment.

\section{Figure 6}

Cultured $\mathrm{HI}$ keratinocytes carrying $A B C A 12$ mutations showed abnormal congestion of lipid, and this phenotype was recovered by corrective $A B C A 12$ gene transfer. (A-D) HI keratinocytes cultured in high $\mathrm{Ca}^{2+}$ conditions showed that glucosylceramide, a major component of LG lipid, was distributed densely around the nuclei (in a congested pattern) (green, FITC). Control normal human keratinocytes showed a widely distributed, diffuse glucosylceramide staining pattern. (E) Electron microscopic (EM) observation revealed, in cultured HI keratinocytes, that apparently amorphous, electron lucent LG-like structures (arrows) formed, but were not secreted. (F) Normal secretion of LG contents (arrow) in a control keratinocyte. (G-O) Before genetic correction, an $\mathrm{HI}$ patient cell showed weak $\mathrm{ABCA} 12$ immunostaining (red, TRITC) and a congested pattern of glucosylceramide staining (green, FITC) (J-L). After genetic correction, an HI patient cell demonstrated stronger ABCA12 labeling (red) and a normal distribution pattern of glucosylceramide (green) (M-O), similar to those of a normal human keratinocyte $(\mathbf{G}-\mathbf{I})$. (P) Corrective gene transfer resulted in a statistically significant increase in the number of cells with completely normal, widely distributed glucosylceramide patterns. ${ }^{*} P<0.02$, Student's $t$ test. Scale bars: $10 \mu \mathrm{m}(\mathbf{A}-\mathbf{D}, \mathbf{G}-\mathbf{0}) ; 0.5 \mu \mathrm{m}(\mathbf{E}, \mathbf{F})$.

\section{Methods}

Patients and families. Four HI patients, patients 1-4, and 1 affected fetus, fetus 1 ( 4 males, 1 female), from 4 independent families, families A-D, showed a serious collapse in the keratinized skin barrier (Figure 1). Family A and family $\mathrm{D}$ were consanguineous (marriage of first cousins). All the patients showed severe hyperkeratosis at birth and presented with generalized, thick scales over their entire body surface with the presence of marked fissuring. Severe ectropion, eclabium, and malformed pinnae were apparent in all cases. Patient 2 from family B died 3 days after birth. Patient 3 from family $\mathrm{C}$ died 15 days after birth, and an affected fetus from family $\mathrm{C}$ was terminated at the parents' request after a positive prenatal diagnostic skin test (33). After written, informed consent was obtained, blood samples were collected from each participating family member, and skin biopsy or autopsy specimens were obtained from the patients and the affected fetus. The study was given appropriate ethical approval by the Ethics Committee at Hokkaido University Graduate School of Medicine.

Mutation screening. Mutation analysis was performed in patients, an affected fetus, and other family members in the 4 families, as far as DNA samples would allow. Briefly, genomic DNA isolated from peripheral blood was subjected to PCR amplification, followed by direct automated sequencing using an ABI PRISM 3100 genetic analyzer (Applied Biosystems). Oligonucleotide primers and PCR conditions used for amplification of exons 1-53 of ABCA12 were originally derived from the report by Lefevre et al. (8) and were partially modified for the present study. The entire coding region, including the exon/intron boundaries for both forward and reverse strands from all patients, family members, and controls, were sequenced. No mutations were found in 100 control alleles from the Japanese population.

Verification of the splice acceptor site mutation. In order to verify the splice acceptor site mutation IVS23-2A $\rightarrow$ G, RT-PCR amplification of mRNA spanning the exon 23-24 boundary was performed using mRNA samples from cultured HI keratinocytes (see below). After RT-PCR amplification, direct sequencing of the products was performed.

Establishment of HI keratinocyte cell culture. A skin sample from patient 1 was processed for primary keratinocyte culture, and cells were grown according to standard procedures in defined keratinocyte serum-free medium (Invitrogen Corp.). Cultures were grown for several passages in low- $\mathrm{Ca}^{2+}(0.09 \mathrm{mM})$ conditions and then switched to high- $\mathrm{Ca}^{2+}(2.0 \mathrm{mM})$ conditions.

RT-PCR of ABCA12 mRNA. RT-PCR of ABCA12 mRNA was performed with Superscript 2 (Invitrogen Corp.) following the manufacturer's 
instructions. Specific primers for PCR amplification were as follows: forward 5'-GAATTGCAAACTGGAAGGAACTCCC-3' and reverse 5'-GAGTCAGCTAGGATTAGACAGC- 3 '. These primers were used for amplification of a 683-bp fragment around exon 23-24 boundary of normal cDNA.

Real time-PCR. For quantitative analysis of $A B C A 12$ expression levels, total RNA extracted was subjected to real-time RT-PCR using the ABI PRISM 7000 sequence detection system (Applied Biosystems). Specific primers/ probes for human ABCA12, TaqMan Gene Expression Assay (HS00292421; Applied Biosystems) were used. Differences between the mean $C T$ values of $A B C A 12$ and those of GAPDH were calculated as $\triangle C T_{\text {sample }}=C T_{\mathrm{ABCA12}}$ - $C T_{\mathrm{GAPDH}}$ and those of the $\triangle C T$ for the cultured keratinocytes in the low $\mathrm{Ca}^{2+}$ condition as $\left(\triangle C T_{\text {calibrator }}=C T_{\mathrm{ABCA} 12}-C T_{\mathrm{GAPDH}}\right)$. Final results, the sample-calibrator ratio, expressed as $n$-fold differences in $A B C A 12$ expression were determined by 2 -( $\triangle \mathrm{CT}$ sample $-\triangle \mathrm{CT}$ calibrator).

Cloning of ABCA12 and corrective gene transfer of HI keratinocytes. Using human keratinocyte cDNA as a template, overlapping clones of human ABCA12 cDNA were amplified by PCR. A composite full-length cDNA was constructed, subjected to nucleotide sequencing, and subcloned into a pCMV-tag4B vector (Stratagene). The expression plasmid pCMV-tag4B$\mathrm{ABCA} 12$ was transfected into $\mathrm{HI}$ keratinocytes using Lipofectamine reagent (Invitrogen Corp.) according to the manufacturer's recommendation. As a control, pCMV-tag4B was transfected into the cells. The transfected cells and control cells from patient 1 were stained with anti-glucosylceramide antibody, and the number of keratinocytes showing the 3 distinct types of glucosylceramide distribution patterns - congested, intermediate, and widely distributed - were assessed and calculated by 1 observer after viewing under a confocal laser scanning microscope, the Olympus Fluoview, FV300 confocal microscope (Olympus).

Morphological observation. Skin biopsy samples or cultured keratinocytes were fixed in $2 \%$ glutaraldehyde solution, postfixed in $1 \%$ OsO4, dehydrated, and processed for conventional electron microscopic observation.

Antibodies. Polyclonal anti-ABCA12 antibody was raised in rabbits using a 14-amino acid sequence synthetic peptide (residues 2567-2580) derived from the ABCA12 sequence (NM 173076) as the immunogen.
The other primary antibody was mouse monoclonal anti-ceramide antibody (Alexis Biochemicals).

Immunofluorescent labeling. Immunofluorescent labeling was performed on frozen tissue sections and keratinocyte cultures as previously described (35). Fluorescent labeling was performed with FITC-conjugated secondary antibodies, followed by $10 \mu \mathrm{g} / \mathrm{ml}$ propidium iodide (Sigma-Aldrich) to counterstain nuclei. For double labeling, fluorescent labeling was performed with a TRITC-conjugated secondary antibody for the anti-ABCA12 antibody. The stained samples were observed under a confocal laser scanning microscope.

Postembedding immunogold electron microscopy. Normal human skin samples were obtained from skin surgical operations under fully informed consent and were processed for postembedding immunoelectron microscopy as previously described (36). Cyrofixed, cryosubstituted samples were embedded in Lowicryl K11M resin (Electron Microscopy Sciences). Ultrathin sections were cut and incubated with anti-ABCA12 antibody, a secondary linker antibody, and a 5-nm gold-conjugated antibody for immunogold labeling.

\section{Acknowledgments}

We thank members of the patients' families for their cooperation; Peter M. Elias for helpful discussion; Sadae Egawa, Haruhiko Sago, Judith Allanson, and Linlea Armstrong for their help; and Akari Nagasaki and Megumi Sato for their technical assistance. This work was supported in part by a Grant-in-Aid from the Ministry of Education, Science, Sports, and Culture of Japan (Kiban B 16390312; to M. Akiyama).

Received for publication February 23, 2005, and accepted in revised form April 12, 2005.

Address correspondence to: Masashi Akiyama, Department of Dermatology, Hokkaido University Graduate School of Medicine, N15 W 7, Sapporo 060-8638, Japan. Phone: 81-11-716-1161; Fax: 81-11-706-7820; E-mail: akiyama@med.hokudai.ac.jp.
1. Williams, M.L., and Elias, P.M. 1987.Genetically transmitted, generalized disorders of cornification; the ichthyoses. Dermatol. Clin. 5:155-178.

2. Judge, M.R., McLean, W.H.I., and Munro, C.S. 2004. Disorders of keratinization. In Rook's textbook of dermatology. 7th edition. T. Burns, S. Breathnach, N. Cox, and C. Griffiths, editors. Blackwell Publishers. Oxford, United Kingdom. 34.1-34.111.

3. Allikmets, R., Gerrard, B., Hutchinson, A., and Dean, M. 1996. Characterization of the human $\mathrm{ABC}$ superfamily: isolation and mapping of 21 new genes using the expressed sequence tags database. Hum. Mol. Genet. 5:1649-1655.

4. Dean, M., Rzhetsky, A., and Allikmets, R. 2001. The human ATP-binding cassette (ABC) transporter superfamily. Genome Res. 11:1156-1166.

5. Borst, P., and Elferink, R.O. 2002. Mammalian $\mathrm{ABC}$ transporters in health and disease. Annu. Rev. Biochem. 71:537-592.

6. Peelman, F., et al. 2003. Characterization of the ABCA transporter subfamily: identification of prokaryotic and eukaryotic members, phylogeny and topology. J. Mol. Biol. 325:259-274.

7. Ishida-Yamamoto, A., et al. 2004. Epidermal lamellar granules transport different cargoes as distinct aggregates. J. Invest. Dermatol. 122:1137-1144.

8. Lefèvre, C., et al. 2003. Mutations in the transporter ABCA12 are associated with lamellar ichthyosis type 2. Hum. Mol. Genet. 12:2369-2378.

9. Vielhaber, G., et al. 2001. Localization of ceramide and glucosylceramide in human epidermis by immunogold electron microscopy. J. Invest. Dermatol. 117:1126-1136.
10. Holleran, W.M., et al. 1993. Processing of epidermal glucosylceramides is required for optimal mammalian cutaneous permeability barrier function. J. Clin. Invest. 91:1656-1664.

11. Dale, B.A., et al. 1990. Heterogeneity in harlequin ichthyosis, an inborn error of epidermal keratinization: variable morphology and structural protein expression and a defect in lamellar granules. J. Invest. Dermatol. 94:6-18.

12. Milner, M.E., O'Guin, W.M., Holbrook, K.A., and Dale, B.A. 1992. Abnormal lamellar granules in harlequin ichthyosis. J. Invest. Dermatol. 99:824-829.

13. Higgins, C.F. 1992. ABC transporters: from microorganisms to man. Annu. Rev. Cell Biol. 8:67-113.

14. Klein, I., Sarkadi, B., and Varadi, A. 1999. An inventory of the human ABC proteins. Biochim. Biophys. Acta. 1461:237-262.

15. Allikmets, R., et al. 1997. A photoreceptor cellspecific ATP-binding transporter gene (ABCR) is mutated in recessive Stargardt macular dystrophy. Nat. Genet. 15:236-246.

16. Allikmets, R., et al. 1997. Mutation of the Stargardt disease gene (ABCR) in age-related macular degeneration. Science. 277:1805-1807.

17. Brooks-Wilson, A., et al. 1999. Mutations in ABC1 in Tangier disease and familial high-density lipoprotein deficiency. Nat. Genet. 22:336-345.

18. Rust, S., et al. 1999. Tangier disease is caused by mutations in the gene encoding ATP-binding cassette transporter 1. Nat. Genet. 22:352-355.

19. Oram, J.F. 2002. Molecular basis of cholesterol homeostasis: lessons from Tangier disease and ABCA1. Trends Mol. Med. 8:168-173.
20. Allikmets, R. 2000. Simple and complex ABCR: genetic predisposition to retinal disease. Am.J. Hum. Genet. 67:793-799.

21. Lewis, R.A., et al. 1999. Genotype-phenotype analysis of a photoreceptor-specific ATP-binding cassette transporter gene, ABCR, in Stargardt disease. Am. J. Hum. Genet. 64:422-434.

22. Huang, W., et al. 2001. Novel mutations in ABCA1 gene in Japanese patients with Tangier disease and familial high density lipoprotein deficiency with coronary heart disease. Biochim. Biophys. Acta 1537:71-78.

23. Hayden, M.R., et al. 2000. Cholesterol efflux regulatory protein, Tangier disease and familial highdensity lipoprotein deficiency. Curr. Opin. Lipidol. 11:117-122.

24. Orso, E., et al. 2000. Transport of lipids from Golgi to plasma membrane is defective in Tangier disease patients and Abc1-deficient mice. Nat. Genet. 24:192-196.

25. Schmitz, G., and Langmann, T. 2001. Structure, function and regulation of the $\mathrm{ABC} 1$ gene product. Curr. Opin. Lipidol. 12:129-140.

26. Weng, J., et al. 1999. Insights into the function of Rim protein in photoreceptors and etiology of Stargardt's disease from the phenotype in abcr knockout mice. Cell. 98:13-23.

27. Klucken, J., et al. 2000. ABCG1 (ABC8), the human homolog of the Drosophila white gene, is a regulator of macrophage cholesterol and phospholipid transport. Proc. Natl. Acad. Sci. U. S. A. 97:817-822.

28. Kaminski, W.E., et al. 2001. Complete coding sequence, promoter region, and genomic structure 
of the human ABCA2 gene and evidence for steroldependent regulation in macrophages. Biochem. Biophys. Res. Commun. 281:249-258.

29. Yamano, G., et al. 2001. ABCA3 is a lamellar body membrane protein in human lung alveolar type II cells. FEBS Lett. 508:221-225.

30. Annilo, T., et al. 2002. Identification and characterization of a novel ABCA subfamily member, ABCA12, located in the lamellar ichthyosis region on 2q34. Cytogenet. Genome Res. 98:169-176.

31. Blanchet-Bardon, C., et al. 1983. Prenatal diagnosis of harlequin fetus [letter]. Lancet. 1:132.
32. Akiyama, M., Kim, D.-K., Main, D.M., Otto, C.E., and Holbrook, K.A. 1994. Characteristic morphologic abnormality of harlequin ichthyosis detected in amniotic fluid cells. J. Invest. Dermatol. 102:210-213.

33. Akiyama, M., Suzumori, K., and Shimizu, H. 1999. Prenatal diagnosis of harlequin ichthyosis by the examinations of keratinized hair canals and amniotic fluid cells at 19 weeks' estimated gestational age. Prenat. Diagn. 19:167-171.

34. Tsuji-Abe, Y., et al. 2004. DNA-based prenatal exclusion of bullous congenital ichthyosiform erythroderma at the early stage, $10-11$ weeks' of pregnancy in two consequent siblings. J. Am. Acad. Dermatol. 51:1008-1011.

35. Akiyama, M., et al. 1999. Periderm cells form cornified cell envelope in their regression process during human epidermal development. J. Invest. Dermatol. 112:903-909.

36. Shimizu, H., McDonald, J.N., Kennedy, A.R., and Eady, R.A.J. 1989. Demonstration of intra- and extra-cellular localization of bullous pemphigoid antigen using cryofixation and freeze substitution for postembedding immuno-electron microscopy. Arch. Dermatol. Res. 281:443-448. 